\title{
The use of technology in tracking soccer players' health performance: a scoping review
}

Jassim Almulla ${ }^{1,2^{*}} \mathbb{D}^{\dagger}$, Abdulrahman Takiddin ${ }^{1,3+}$ and Mowafa Househ ${ }^{1}$

\begin{abstract}
Background: Quantifying soccer players' performance using different types of technologies helps coaches in making tactical decisions and maintaining players' health. Little is known about the relation between the performance measuring technologies and the metrics they measure. The aim of this study is to identify and group the different types of technologies that are used to track the health-related performance metrics of soccer players.

Methods: We conducted a systematic search for articles using IEEE Xplore, PubMed, ACM DL, and papers from the Sports Medicine Journal. The papers were screened and extracted by two reviewers. The included papers had to fall under several criteria, including being about soccer, measuring health-related performance, and using technology to measure players' performance. A total of 1,113 papers were reviewed and 1,069 papers were excluded through the selection process.

Results: We reviewed 44 papers and grouped them based on the technology used and health-related metrics tracked. In terms of technology, we categorized the used technologies into wearable technologies $(\mathrm{N}=27 / 44)$ and in-field technologies ( $N=14 / 44)$. We categorized the tracked health-related metrics into physiological metrics ( $N=16 / 44)$ and physical metrics $(N=44 / 44)$. We found out that wearable technologies are mainly used to track physical metrics ( $N=27 / 27)$ and are also used to track physiological metrics ( $N=14 / 27)$. In-field technologies are only used to track physical metrics ( $N=24 / 24)$.

Conclusion: Understanding how technology is related to players' performance and how it is used leads to an improvement in the monitoring process and performance outcomes of the players.
\end{abstract}

Keywords: Soccer, Player, Health, Performance, Technology

\section{Background}

As soccer is gaining popularity around the world, soccer organizations and researchers are attempting to improve the overall performance of the players, maintain their health, and win more matches [1]. The performance of the players is being tracked in official matches as part of local or international competitions [2], and in to unofficial

\footnotetext{
*Correspondence: jmalmulla@hbku.edu.qa

† Jassim Almulla and Abdulrahman Takiddin contributed equally to this paper. ${ }^{1}$ College of Science and Engineering, Hamad Bin Khalifa University, Doha, Qatar ${ }^{2}$ Qatar Football Association, Doha, Qatar

Full list of author information is available at the end of the article
}

matches such as team's training sessions, training matches among team players, and friendly matches with other teams $[3,4]$. Official matches tend to be more intense than unofficial matches due to the pressure to win these matches.

Soccer coaches are interested in technical and healthrelated performance metrics [5] as both are essential when it comes to quantifying the overall performance of the players [6,7]. Technical performance metrics include players' activities during the match, such as the number of successful passes, duration of ball possession, number of passes among players, and number of shots the player 
attempts to score a goal $[1,8]$. The health-related performance metrics include the total distance covered by players in moderate, high, and very high speeds [5].

To monitor the players' performance, soccer organizations and researchers are using different types of tracking technologies that are capable of tracking different metrics of the players. These technologies replace the manual process where specialized observers review taped videos of the matches and code players' activity patterns [3]. These technologies help reduce the time needed to collect data about the players during matches and assist coaches in gathering more data about different aspects of the players.

The aim of this study is to identify the different technologies used to track performance metrics of soccer players in recent literature. The study also identifies and categorizes the different health-related performance of soccer players. Identifying and linking the various technologies to health-related metrics help coaches and health practitioners in the sports field to focus on many aspects of the player to maintain players' health conditions, which leads to enhance the overall performance of the players.

\section{Method \\ Search strategy}

We conducted a systematic search and included studies up until the 31st of March, 2019. We identified the articles from IEEE Xplore, PubMed, and ACM DL. In order to have more specialized journals in the sports field, we searched within SJR's (SCImago Journal Rank) list of highly ranked journals under the "Sports Science" category and chose the Sports Medicine Journal, which is one of the most highly ranked international journals. Then, we identified the papers using the "Springer Link" database search engine.

We searched IEEE Xplore, ACM DL, and Springer Link databases using the combination of the following keywords: (1) "soccer", "football"; (2) "technology", "system"; (3) "athlete", "player"; (4) "performance". We connected the terms with the "OR" term within each of the four combination groups. We combined the four combination groups using the "AND" term. The exact phrasing of the search keywords is:

(soccer OR football) AND performance AND
(technology OR system) AND (athlete OR player)

In the Pubmed database, we used the "MeSH" (Medical Subject Headings) approach, which is the national library of medicine's controlled vocabulary thesaurus that is used for indexing and organizing articles. Each article is associated with $\mathrm{MeSH}$ terms that describe the content of the citation. Our MeSH search term included "Soccer" and "Athletic Performance" as two mesh phrases joined by the
"AND" keyword. Then that, we separated the keywords "technology" and "system" by the "OR" keyword. The exact phrasing of the search keywords is:

("Soccer"[Mesh]) AND "Athletic

Performance"[Mesh] (technology OR system)

\section{Selection criteria}

The selection criteria was based on four main requirements. First, the study had to be about soccer only. We used the keyword "football" since it is used to refer to soccer in some countries, and excluded any study that discussed American football, rugby, or futsal (indoor soccer). Second, the study had to focus on players' performance. As such, we excluded studies that assessed the performance of a particular technology. Third, the study had to be about monitoring players' performance through technology. Thus, we excluded studies that monitored players' performance through manual monitoring techniques. Finally, the paper had to be published within the last ten years (i.e. 2009-2019). To visualize our search process, we used the Preferred Reporting Items for Systematic Reviews and Meta-Analyses (PRISMA), as illustrated in Fig. 1.

After completing the basic search, we had a total of $(\mathrm{N}=1113)$ papers. Then, we started excluding papers in three phases. In the first phase, we excluded papers $(\mathrm{N}=380)$ due to the following reasons: non-English papers $(\mathrm{N}=8)$, papers published before $2009(\mathrm{~N}=366)$, and duplicate papers $(\mathrm{N}=6)$.

In the second phase, we, the two assessors (JM an AT), independently assessed a total of $(\mathrm{N}=733)$ papers using the title and abstract of the article. We excluded a number of papers $(\mathrm{N}=644)$ based on the following exclusion criteria. We excluded: (1) response or review papers $(\mathrm{N}=131)$; (2) papers related to sports other than soccer, such as American football, rugby, and futsal $(\mathrm{N}=210)$; (3) papers that used manual performance tracking approaches or papers that did not specify the used performance tracking method $(\mathrm{N}=107)$; (4) papers that were not related to player's performance, such as player's injury, strategy, or recovery $(\mathrm{N}=210)$; and (5) papers that were not related professional players, such as referees and home training $(\mathrm{N}=6)$.

In the last phase, we included a total of 69 papers $(\mathrm{N}=69)$ for full-text review. After reviewing the full text, we excluded some papers $(\mathrm{N}=25)$ based on the following criteria. We excluded: (1) studies that analyzed a whole team, but not individual players within the team $(\mathrm{N}=1)$; (2) papers related to players' technical performance, such as the number of passes $(\mathrm{N}=8)$; (3) studies that used manual performance analysis or used existing datasets without involving technology in the analysis 


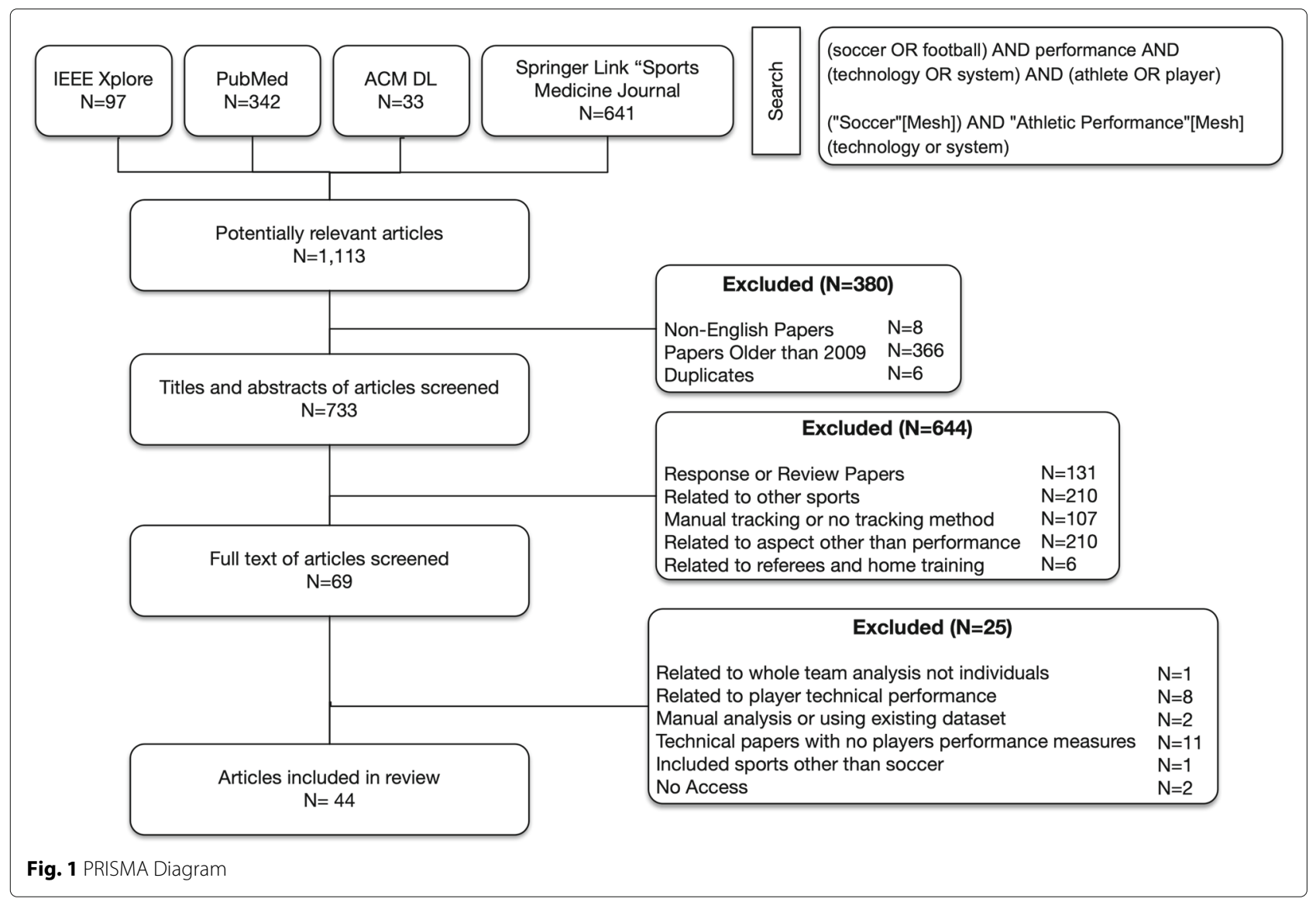

$(\mathrm{N}=2)$; (4) technical papers that did not discuss players' performance metrics $(\mathrm{N}=11)$; (5) studies that did not focus on soccer only but rather discussed other sports $(\mathrm{N}=1)$; and (6) papers that the assessors could not access $(\mathrm{N}=2)$.

\section{Results}

\section{Study characteristics}

Figure 2 shows the number of papers published per year since 2009. We observed that the number of papers was increasing until $2016(\mathrm{~N}=9 / 44)$. Then, the number of published papers dropped in the following two years. We also analyzed the location of the study, as shown in Fig. 3. Around $72 \%(\mathrm{~N}=32 / 44)$ of the studies were conducted in Europe, 16\% ( $\mathrm{N}=7 / 44)$ in Asia-Pacific region, and 2\% $(\mathrm{N}=1 / 44)$ in South America. The rest of the papers did not specify the location of the study $(\mathrm{N}=4 / 44)$.

Table 1 summarizes the sample size, gender, age group, and the study duration of the conducted studies in the papers. The number of participants in the studies varies from 6 to more than 26,000 players. More than 86\% $(\mathrm{N}=38 / 44)$ of the papers experimented with male players while only one paper $(\mathrm{N}=1 / 44)$ studied female players. The rest of the papers $(\mathrm{N}=5 / 44)$ did not specify the gender. The number of matches in the studies ranged from 1 match to 460 matches.

The age of the players is addressed in the papers using 2 representations; standard age range in years and the soccer notation, such as senior (players in the first league) and U23 (under 23 years old). The age of the players in the studies ranges from 9 to 30 years old. We categorized the ages of the participating players into three main groups: (1) Senior: players above 23 years old; (2)

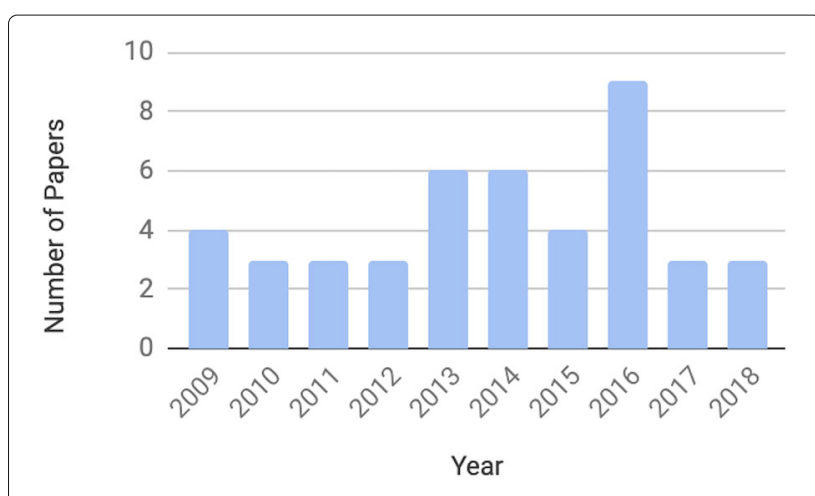

Fig. 2 Number of published papers per year 


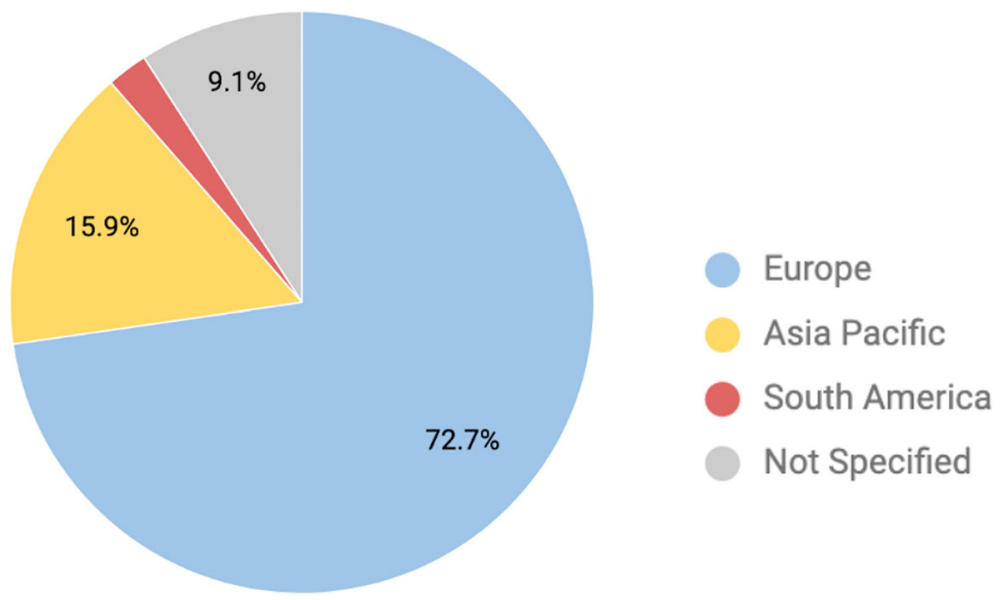

Fig. 3 Study Location per region

Youth: players between 18 and 23 years old; and (3) Young: players below 18 years old. More than $54 \%$ of the papers $(\mathrm{N}=24 / 44)$ focused on senior players, around $23 \%$ of the papers studied young players $(\mathrm{N}=10 / 44)$, and around $20 \%$ of the papers studied youth players $(\mathrm{N}=9 / 44)$. One paper did not specify the age of the studied players $(\mathrm{N}=1 / 44)$.

The duration of the studies ranged from 1 day to 7 soccer seasons. Out of the 44 papers, 34 papers specified the duration of the conducted studies $(\mathrm{N}=34 / 44)$, whereas 10 papers did not specify $(\mathrm{N}=10 / 44)$. We grouped the duration of the studies into three groups: (1) one-day-study: studies conducted in one day $(\mathrm{N}=2 / 34)$; (2) weeks: studies conducted over weeks $(\mathrm{N}=5 / 34)$, which ranged from 3 to 39 weeks; and (3) seasons: studies that lasted for one or more soccer seasons $(\mathrm{N}=24 / 34)$. In the seasons group, $66 \%$ of the papers were conducted during 1 season $(\mathrm{N}=16 / 24)$, and the rest of the papers lasted between 2 and 7 seasons $(\mathrm{N}=8 / 24)$.

\section{Type of match}

We divided the studies into two groups according to the match type used for data collection: studies that were conducted on players during official matches and studies that were conducted on players during unofficial matches. Approximately $91 \%(\mathrm{~N}=40 / 44)$ of the papers specified the match type. Out of the 40 studies, $40 \%(\mathrm{~N}=16 / 40)$ of the papers conducted their experiments with players during official matches, whereas $60 \%(\mathrm{~N}=24 / 40)$ experimented with players during unofficial sessions. The rest of the studies $(\mathrm{N}=4 / 44)$ did not specify the match type of the conducted experiments.

\section{Tracking technologies}

We extracted different types of technologies used to track the players during matches. Global Positioning System (GPS) is used to measure the position of the players for each second in time [1,9]. Local Position Measurement System (LPMS) is a system that constantly tracks the players' body movements and acceleration [4]. Heart Rate Monitor (HRM) is a device that is used to monitor the heart rate of the players and associate it with different actions and events [10]. Accelerometer (ACC) is a motor sensor that quantifies the physical activities and physiological demands of the players; it is highly responsive to the acceleration of the body movements and records it in three dimensions [20]. Gyroscope (GS), Digital Compass (DC), and Microelectromechanical System (MEMS) are components within the GPS that are used to improve the accuracy of the readings $[11,44]$. In addition to these technologies, sensors, which are devices attached to the players, are used to transfer the collected data by microwave radio channels to other sensors mounted on the soccer arena [20]. Also, the Multi-Camera System (MCS) consists of cameras mounted on the stadium's roof and the footage is analyzed using proprietary software [12, 23].

As shown in Table 2, we divided all the performance monitoring technologies into two groups. First, wearable technologies include the devices that are attached to the players to monitor their performance. Approximately $68 \%$ of the papers $(\mathrm{N}=30 / 40)$ used wearable technologies to track the performance of the players. Approximately $90 \%$ of those studies $(\mathrm{N}=27 / 30)$ used GPS technology. The other wearable technologies are GS, DC, MEMS, LPMS, HRM, ACC, and sensors, which complement the GPS to get more specific and accurate data about the players. Second, in-field technologies include the devices that are installed in the soccer arena to monitor the players. Approximately $39 \%$ of the studies $(\mathrm{N}=24 / 44)$ used in-field technology to track the performance of the players. The in-field technology refers to the MCS. Only $9 \%$ of the studies $(\mathrm{N}=4 / 44)$ used both wearable and in-field technologies to track the performance of the players. 
Table 1 Summary findings

\begin{tabular}{|c|c|c|c|c|c|}
\hline Reference & Sample Size & Gender & Age Group & Duration & Matches \\
\hline (Ric et al. 2017) [1] & 19 & M & Senior & $1 \mathrm{D}$ & 1 \\
\hline (Wehbe et al. 2014) [2] & 19 & M & Senior & $1 \mathrm{~S}$ & 8 \\
\hline (Malone et al, 2018) [5] & 48 & M & Senior & $1 \mathrm{~S}$ & 460 \\
\hline (Scott et al. 2013) [6] & 15 & M & Senior & $1 \mathrm{~S}$ & 29 \\
\hline (Moalla et al. 2018) [8] & 17 & $x$ & Senior & $2 S$ & 52 \\
\hline (Akenhead et al. 2016) [44] & 33 & M & Senior & $39 W$ & 195 \\
\hline (Castagna et al. 2017) [9] & 1,200 & M & Senior & $1 \mathrm{~S}$ & 60 \\
\hline (Torreño et al. 2016) [10] & 26 & M & Senior & $1 \mathrm{~S}$ & $x$ \\
\hline (Rossi et al. 2016) [11] & 26 & M & Senior & $1 \mathrm{~S}$ & 80 \\
\hline (Bradley et al. 2014) [12] & 810 & M & Senior & $1 \mathrm{~S}$ & 20 \\
\hline (Di Mascio and Bradley 2013) [13] & 100 & M & Senior & $1 \mathrm{~S}$ & 20 \\
\hline (Carling et al. 2016) [14] & 12 & M & Senior & $1 \mathrm{~S}$ & 31 \\
\hline (Ingebrigtsen et al. 2015) [15] & 15 & M & Senior & $1 \mathrm{~S}$ & 15 \\
\hline (Varley and Aughey 2013) [16] & 29 & M & Senior & $1 \mathrm{~S}$ & 34 \\
\hline (Vigne et al. 2010) [17] & 25 & M & Senior & $1 \mathrm{~S}$ & 30 \\
\hline (Andrzejewski et al. 2012) [18] & 31 & M & Senior & $1 \mathrm{~S}$ & 4 \\
\hline (Castellano et al. 2011) [19] & 434 & M & Senior & $1 \mathrm{~S}$ & $x$ \\
\hline (Dalen et al. 2016) [20] & 45 & M & Senior & 35 & $x$ \\
\hline (Bradley et al. 2013) [21] & 711 & M & Senior & $3 S$ & $x$ \\
\hline (Di Salvo et al. 2009) [22] & 563 & M & Senior & $3 S$ & $x$ \\
\hline (Di Salvo et al. 2012) [23] & 26,449 & M & Senior & $4 S$ & $x$ \\
\hline (Bradley et al. 2016) [24] & $x$ & M & Senior & $7 S$ & 1 \\
\hline (Bush et al. 2015) [25] & 1,036 & M & Senior & $7 S$ & $x$ \\
\hline (Rampinini et al. 2009) [26] & 186 & M & Senior & $x$ & 416 \\
\hline (Randers et al. 2010) [3] & 20 & $x$ & Youth & $1 \mathrm{D}$ & 1 \\
\hline (Stevens T et al. 2014) [4] & 12 & M & Youth & $x$ & $x$ \\
\hline (Russell et al. 2016) [27] & 11 & M & Youth & $1 \mathrm{~S}$ & 19 \\
\hline (Casamichana et al. 2012) [28] & 27 & M & Youth & $2 \mathrm{~S}$ & $x$ \\
\hline (Hodgson et al. 2014) [29] & 8 & M & Youth & $4 S$ & 3 \\
\hline (Mugglestone et al. 2013) [30] & 20 & M & Youth & $x$ & 50 \\
\hline (Bendiksen et al. 2013) [31] & 11 & $\mathrm{~F}$ & Youth & $x$ & $x$ \\
\hline (Varley et al. 2017) [32] & 6 & $x$ & Youth & $x$ & $x$ \\
\hline (Harley et al. 2011) [33] & 6 & M & Youth & $x$ & $x$ \\
\hline (Coutinho et al. 2018) [7] & 90 & M & Young & $x$ & $x$ \\
\hline (Fernandes-da-Silva et al. 2016) [34] & 33 & M & Young & $3 W$ & 9 \\
\hline (Hill-Haas et al. 2009) [35] & 16 & M & Young & $9 \mathrm{~W}$ & $x$ \\
\hline (Abade et al. 2014) [36] & 151 & M & Young & $9 \mathrm{~W}$ & 38 \\
\hline (Abade et al. 2014) [37] & 151 & M & Young & $1 \mathrm{~S}$ & 38 \\
\hline (Goto et al. 2015) [38] & 34 & $x$ & Young & $2 S$ & $x$ \\
\hline (Buchheit et al. 2010) [39] & 77 & M & Young & $8 S$ & $x$ \\
\hline (Castagna et al. 2009) [40] & 21 & M & Young & $x$ & $x$ \\
\hline (Goto et al. 2015) [41] & 81 & M & Young & $x$ & $x$ \\
\hline (Buchheit et al. 2011) [42] & 104 & $x$ & Young & $x$ & 66 \\
\hline (Nagahara et al. 2016) [43] & 20 & M & $x$ & $x$ & 2 \\
\hline
\end{tabular}

Legend: $\boldsymbol{X}=$ Information Not Available, $\mathrm{M}=$ Male, $\mathrm{F}=$ Female, $\mathrm{D}=$ Day, $\mathrm{W}=$ Week, $\mathrm{S}=$ Season 
Table 2 Players' performance tracking technologies in soccer

\begin{tabular}{lll}
\hline Category & Technology & Sources \\
\hline Wearable & GPS & {$[1-7,9-11,16,27-30,32-39,41-44]$} \\
& GS & {$[11]$} \\
& DC & {$[11]$} \\
& MEMS & {$[44]$} \\
& LPMS & {$[4]$} \\
& HRM & {$[5,6,10,35-37,40]$} \\
& ACC & {$[5,6,10,11,20,28,30,37]$} \\
& Sensor & {$[15,20,31]$} \\
In-Field & MCS & {$[1-7,10,11,15,16,28-31,34-37$,} \\
& & $39-41,43,44]$
\end{tabular}

\section{Performance metrics}

Different health-related metrics were extracted from the papers. The Heart Rate (HR) of the players is usually measured in predefined time intervals [35]. The Metabolic Power (MP) is a method that shows the soccer player's specific activity using speed and acceleration [9]. Muscular Fatigue (MSF) is when the muscles of players are unable to generate force [7]. The Distance Covered (DC) is the quantified distance that each player covers during the match. Speed (SP) refers to the running speed of the players. Acceleration and Deceleration (AD) refer to the change of speed of soccer players during a match.

We categorized the performance metrics into two main categories, as shown in Table 3. The first category is the physiological metrics, which includes the metrics that are related to the functions of the players' internal organs. The physiological metrics are the HR, MP, and MSF. Approximately $36 \%(\mathrm{~N}=16 / 44)$ of the papers studied the physiological metrics of the players, $69 \%(\mathrm{~N}=11 / 16)$ of those papers focused specifically on HR, four papers discussed MP $(\mathrm{N}=4 / 16)$, and two papers discussed MSF $(\mathrm{N}=2 / 16)$. The second category is the physical metrics, which includes metrics related to the physical activity of the players during the soccer match. The physical

Table 3 Soccer players' performance metrics

\begin{tabular}{lll}
\hline Category & Performance Metric & Sources \\
\hline Physiological & HR & {$[5,6,10,29,31,34-37,40,44]$} \\
& MP & {$[4,9,11,29]$} \\
& MSF & {$[7,11]$} \\
Physical & DC & {$[5,6,8-12,20,24-26,29,33,34,36$,} \\
& & $40]$ \\
& SP & {$[1-10,12,13,15-44]$} \\
& AD & {$[2,4,7,9-11,15,16,20,27,32,37$,} \\
& & $39,44]$ \\
\hline
\end{tabular}

metrics are the DC, SP, and AD. All of the studied papers $(\mathrm{N}=44 / 44)$ discussed the physical performance of the players.

\section{Relationship between findings}

Table 4 summarizes and links the studies according to the match type, technology type, and the performance measured. Out of the studies conducted during official matches $(\mathrm{N}=16 / 40)$, most of them $(\mathrm{N}=13 / 16)$ used infield technologies only, one study $(\mathrm{N}=1 / 16)$ used wearable technologies, and the remaining $(\mathrm{N}=2 / 16)$ used both wearable and in-field technologies to measure the performance of the players. Out of the studies conducted during unofficial matches $(\mathrm{N}=24 / 40)$, around $91 \%$ of them $(\mathrm{N}=22 / 24)$ used only wearable technologies and the rest $(\mathrm{N}=2 / 24)$ used both wearable and in-field technologies as their tracking methods. In $61 \%$ of the papers $(\mathrm{N}=27 / 44)$, wearable technologies were used to track the performance metrics. Wearable technologies were used to track both physical and physiological performance. Wearable technologies were used to track players' physical performance in all papers $(\mathrm{N}=27 / 27)$. Wearable technologies were also used in $52 \%$ of the studies $(\mathrm{N}=14 / 27)$ to track physiological performance. On the other hand, in $32 \%$ of the studies $(\mathrm{N}=14 / 44)$, in-field technologies were used to track the physical performance of the players only, whereas the physiological metrics were never tracked using in-field technologies.

A few papers $(\mathrm{N}=4 / 44)$ used both wearable and in-field technologies to track the performance of the players. In two studies [3, 33], the authors compared the outcomes of wearable and in-field technologies. In another study [4], the wearable technologies were the main tracking method used for the study and the in-field technology was used as a gold standard, which means that it was used just to confirm the results.

\section{Discussion}

Our paper summarized the findings in the literature to identify and group the different health-related performance metrics tracked for soccer players along with the technologies used to track these metrics in official and unofficial matches. All of the papers we reviewed conducted experiments with soccer players using different technologies to assess different performance aspects without relating the use of technology to the performance aspects. Our contribution in this paper filled this gap by linking each technology used with the performance metrics it measures. We also identified the type of match that the technology is used in. To the best of our knowledge, no similar work has been done to fill this gap.

Based on this review, there were only three papers that studied the performance of soccer players in official matches using wearable technologies. These studies were conducted in 2016 and 2017. In 2015, the International 
Table 4 Technology types used in matches

\begin{tabular}{|c|c|c|c|c|c|}
\hline \multirow[t]{2}{*}{ Source } & \multirow[t]{2}{*}{ Match Type } & \multicolumn{2}{|c|}{ Technology Type } & \multicolumn{2}{|c|}{ Performance Measured } \\
\hline & & Wearable & In-Field & Physiological & Physical \\
\hline (Castagna et al. 2017) [9] & Official & $\checkmark$ & $\checkmark$ & $x$ & $\checkmark$ \\
\hline (Dalen et al. 2016) [20] & Official & $\checkmark$ & $\checkmark$ & $x$ & $\checkmark$ \\
\hline (Moalla et al. 2018) [8] & Official & $x$ & $\checkmark$ & $x$ & $\checkmark$ \\
\hline (Bradley et al. 2014) [12] & Official & $x$ & $\checkmark$ & $x$ & $\checkmark$ \\
\hline (Di Mascio and Bradley 2013) [13] & Official & $x$ & $\checkmark$ & $x$ & $\checkmark$ \\
\hline (Carling et al. 2016) [14] & Official & $x$ & $\checkmark$ & $x$ & $\checkmark$ \\
\hline (Vigne et al. 2010) [17] & Official & $x$ & $\checkmark$ & $x$ & $\checkmark$ \\
\hline (Andrzejewski et al. 2012) [18] & Official & $x$ & $\checkmark$ & $x$ & $\checkmark$ \\
\hline (Castellano et al. 2011) [19] & Official & $x$ & $\checkmark$ & $x$ & $\checkmark$ \\
\hline (Bradley et al. 2013) [21] & Official & $x$ & $\checkmark$ & $x$ & $\checkmark$ \\
\hline (Di Salvo et al. 2009) [22] & Official & $x$ & $\checkmark$ & $x$ & $\checkmark$ \\
\hline (Di Salvo et al. 2012) [23] & Official & $x$ & $\checkmark$ & $x$ & $\checkmark$ \\
\hline (Bradley et al. 2016) [24] & Official & $x$ & $\checkmark$ & $x$ & $\checkmark$ \\
\hline (Bush et al. 2015) [25] & Official & $x$ & $\checkmark$ & $x$ & $\checkmark$ \\
\hline (Rampinini et al. 2009) [26] & Official & $x$ & $\checkmark$ & $x$ & $\checkmark$ \\
\hline (Russell et al. 2016) [27] & Official & $\checkmark$ & $x$ & $x$ & $\checkmark$ \\
\hline (Ric et al. 2017) [1] & Unofficial & $\checkmark$ & $x$ & $x$ & $\checkmark$ \\
\hline (Wehbe et al. 2014) [2] & Unofficial & $\checkmark$ & $x$ & $x$ & $\checkmark$ \\
\hline (Ingebrigtsen et al. 2015) [15] & Unofficial & $\checkmark$ & $x$ & $x$ & $\checkmark$ \\
\hline (Varley and Aughey 2013) [16] & Unofficial & $\checkmark$ & $x$ & $x$ & $\checkmark$ \\
\hline (Mugglestone et al. 2013) [30] & Unofficial & $\checkmark$ & $x$ & $x$ & $\checkmark$ \\
\hline (Buchheit et al. 2010) [39] & Unofficial & $\checkmark$ & $x$ & $x$ & $\checkmark$ \\
\hline (Goto et al. 2015) [41] & Unofficial & $\checkmark$ & $x$ & $x$ & $\checkmark$ \\
\hline (Nagahara et al. 2016) [43] & Unofficial & $\checkmark$ & $x$ & $x$ & $\checkmark$ \\
\hline (Malone et al, 2018) [5] & Unofficial & $\checkmark$ & $x$ & $\checkmark$ & $\checkmark$ \\
\hline (Scott et al. 2013) [6] & Unofficial & $\checkmark$ & $x$ & $\checkmark$ & $\checkmark$ \\
\hline (Coutinho et al. 2018) [7] & Unofficial & $\checkmark$ & $x$ & $\checkmark$ & $\checkmark$ \\
\hline (Akenhead et al. 2016) [44] & Unofficial & $\checkmark$ & $x$ & $\checkmark$ & $\checkmark$ \\
\hline (Torreño et al. 2016) [10] & Unofficial & $\checkmark$ & $x$ & $\checkmark$ & $\checkmark$ \\
\hline (Rossi et al. 2016) [11] & Unofficial & $\checkmark$ & $x$ & $\checkmark$ & $\checkmark$ \\
\hline (Casamichana et al. 2012) [28] & Unofficial & $\checkmark$ & $x$ & $\checkmark$ & $\checkmark$ \\
\hline (Hodgson et al. 2014) [29] & Unofficial & $\checkmark$ & $x$ & $\checkmark$ & $\checkmark$ \\
\hline (Bendiksen et al. 2013) [31] & Unofficial & $\checkmark$ & $x$ & $\checkmark$ & $\checkmark$ \\
\hline (Fernandes-da-Silva et al. 2016) [34] & Unofficial & $\checkmark$ & $x$ & $\checkmark$ & $\checkmark$ \\
\hline (Hill-Haas et al. 2009) [35] & Unofficial & $\checkmark$ & $x$ & $\checkmark$ & $\checkmark$ \\
\hline (Abade et al. 2014) [36] & Unofficial & $\checkmark$ & $x$ & $\checkmark$ & $\checkmark$ \\
\hline (Castagna et al. 2009) [40] & Unofficial & $\checkmark$ & $x$ & $\checkmark$ & $\checkmark$ \\
\hline (Abade et al. 2014) [37] & Unofficial & $\checkmark$ & $x$ & $\checkmark$ & $\checkmark$ \\
\hline (Randers et al. 2010) [3] & Unofficial & $\checkmark$ & $\checkmark$ & $x$ & $\checkmark$ \\
\hline (Stevens T et al. 2014) [4] & Unofficial & $\checkmark$ & $\checkmark$ & $\checkmark$ & $\checkmark$ \\
\hline (Varley et al. 2017) [32] & $x$ & $\checkmark$ & $x$ & $x$ & $\checkmark$ \\
\hline (Harley et al. 2011) [33] & $x$ & $\checkmark$ & $x$ & $x$ & $\checkmark$ \\
\hline (Goto et al. 2015) [38] & $x$ & $\checkmark$ & $x$ & $x$ & $\checkmark$ \\
\hline (Buchheit et al. 2011) [42] & $x$ & $\checkmark$ & $x$ & $x$ & $\checkmark$ \\
\hline
\end{tabular}

Legend: $\checkmark=$ Measured, $\boldsymbol{x}=$ Not Measured 
Football Association Board (IFAB) approved the use of wearable technologies in official matches [45]. Before 2015, in-field technologies were the main method to monitor the performance of the players in official matches, whereas wearable technologies were used in unofficial matches. In-field technologies are not usually used during unofficial matches due to the high cost of operation and the need for a specialized operator to run the system [3]. Also, in-field technologies require special installation in the club's main stadium, where only some matches take place [33]. Training sessions and unofficial matches are sometimes conducted in training fields where in-field technologies are not available. For that reason, researchers and team officials rely on wearable technologies to track the performance of players during training sessions and unofficial matches as it is portable and relatively easier to operate.

In-field technologies are only used to track the physical performance of the players as MCS can automatically track the CD, SP, and AD of the players. However, studying the physiological aspects of the players has to be done through direct interaction with the player's body, which cannot be achieved using the in-field multi-cameras system. The wearable technologies are used to track all different types of health-related performance metrics required by the researchers. There are a few papers that used both types of technologies in their studies. However, their main purpose was to compare the results of both technologies.

The physical metrics were tracked by all the papers in our study. We discovered that there are three main physical metrics that were tracked in the papers: (1) distance covered, (2) speed, and (3) acceleration and deceleration. Tracking the physical performance is crucial since it is directly related to the performance of the team during a match, which also affects the technical performance of the players and eventually winning a game. Both in-field and wearable technologies were used to track the physical performance of the players. Mainly, wearable technologies were used to track the players' physical performance during unofficial matches, whereas in-field technologies were used to track the physical performance during official matches.

The physiological performances of the players were tracked using wearable devices only in the studied papers. We identified three main physiological metrics: (1) HR, (2) MP, and (3) MSF. All the included studies that analyzed physiological performance were on unofficial matches. We believe that more work has to be done regarding the physiological performance during official matches since players tend to have higher intensity activities compared to unofficial matches. Studying the physiological performance during official matches will allow coaches and researchers to understand and improve players' performance during official matches accordingly.
Most of the studied papers focused on the physical performance of the players in the first place, which is due to the ease of measuring the physical performance using the different types of technologies. Also, monitoring the physical performance of the players does not require special skills to analyze and understand the readings by coaches, players, and technical staff. However, monitoring the physiological performance requires more specialized practitioners and needs specific skills to interpret the results.

\section{Limitation}

This scoping review examined papers written in English; other languages were not included, which might have excluded some studies conducted in other parts of the world like South America. A limitation might be the gap between the time the research was done and the time the work was submitted, which will exclude published papers during that period. We conducted a systematic search in the academic databases to include all published papers in this area, but we might have missed some. Although we tried to discuss all the findings in the literature, it is impossible to detail all the findings found in the papers.

\section{Future work}

There is a need to compare the performance of the in-field technologies with the wearable technologies to track the physical performance of the players. Also, future research should focus more on the physiological aspects of the players as it helps in understanding the players' health status. More studies are required on female players due to the lack of studies conducted on them compared to male players. It is also important to link the monitored metrics to the health of the players.

\section{Conclusion}

Technology has automated the process of measuring soccer players' performance. Two types of technologies are used to monitor health-related performances. Players' physiological performances are determined primarily using wearable technologies. The physical performance of the player is measured using both wearable and in-field technologies. Understanding the relationship between technology and performance as well as how and where it is being used helps in improving the monitoring process, which leads to improving the overall performance of the players.

\section{Abbreviations}

ACC: Accelerometer; AD: Acceleration \& Deceleration; DC: Distance Covered; GPS: Global Positioning System; GS: Gyroscope; HR: Heart Rate; HRM: Heart Rate Monitor; LPMS: Local Position Measurement System; MCS: Multi-Camera System; MEMS: Microelectromechanical System; MeSH: Medical Subject Headings; MP: Metabolic Power; MSF: Muscular Fatigue; PRISMA: Preferred Reporting Items for Systematic Reviews and Meta-Analyses 


\section{Acknowledgements}

Not applicable.

\section{Authors' contributions}

JA and AT performaed the scoping review of the papers equally and have contributed in writing the manuscript equally. $\mathrm{MH}$ has supervised the process and reviewed the final manuscript. All authors read and approved the final manuscript.

\section{Funding}

This study is funded by Qatar National Library "QNL".

\section{Availability of data and material}

The datasets used and/or analysed during the current study are available from the corresponding author on reasonable request.

\section{Ethics approval and consent to participate}

Not applicable.

\section{Consent for publication}

Not applicable.

\section{Competing interests}

The authors declare that they have no competing interests.

\section{Author details}

${ }^{1}$ College of Science and Engineering, Hamad Bin Khalifa University, Doha, Qatar. ${ }^{2}$ Qatar Football Association, Doha, Qatar. ${ }^{3}$ Texas A\&M University at Qatar, Doha, Qatar.

Received: 16 June 2019 Accepted: 16 June 2020

Published online: 11 August 2020

\section{References}

1. Ric A, Torrents C, Gonçalves B, Torres-Ronda L, Sampaio J, Hristovski R. Dynamics of tactical behaviour in association football when manipulating players' space of interaction. PLoS One. 2017;12(7):0180773.

2. Wehbe GM, Hartwig TB, Duncan CS. Movement analysis of australian national league soccer players using global positioning system technology. J Strength Cond Res. 2014;28(3):834-42.

3. Randers MB, Mujika I, Hewitt A, Santisteban J, Bischoff R, Solano R, Zubillaga A, Peltola E, Krustrup P, Mohr M. Application of four different football match analysis systems: a comparative study. J Sports Sci. 2010;28(2):171-82

4. Stevens T GA, de Ruiter CJ, van Niel C, van de Rhee R, Beek PJ, Savelsbergh GJP. Measuring acceleration and deceleration in soccer-specific movements using a local position measurement (LPM) system. Int J Sports Physiol Perform. 2014;9(3):446-56.

5. Malone S, Owen A, Newton M, Mendes B, Tiernan L, Hughes B, Collins K. Wellbeing perception and the impact on external training output among elite soccer players. J Sci Med Sport. 2018;21(1):29-34.

6. Scott BR, Lockie RG, Knight TJ, Clark AC, Janse de Jonge XAK. A comparison of methods to quantify the in-season training load of professional soccer players. Int J Sports Physiol Perform. 2013;8(2): 195-202.

7. Coutinho D, Gonçalves B, Wong DP, Travassos B, Coutts AJ, Sampaio J. Exploring the effects of mental and muscular fatigue in soccer players' performance. Hum Mov Sci. 2018;58:287-96.

8. Moalla W, Fessi MS, Makni E, Dellal A, Filetti C, Di Salvo V, Chamari K. Association of physical and technical activities with partial match status in a soccer professional team. J Strength Cond Res. 2018;32(6):1708-14.

9. Castagna C, Varley M, Póvoas SCA, D'Ottavio S. Evaluation of the match external load in soccer: Methods comparison. Int J Sports Physiol Perform. 2017;12(4):490-5

10. Torreño N, Munguía-Izquierdo D, Coutts A, de Villarreal ES, Asian-Clemente J, Suarez-Arrones L. Relationship between external and internal loads of professional soccer players during full matches in official games using global positioning systems and Heart-Rate technology. Int J Sports Physiol Perform. 2016;11(7):940-6.

11. Rossi A, Perri E, Trecroci A Savino M, Alberti G, laia MF Characterization of in-season elite football trainings by GPS features: The identity card of a
Short-Term football training cycle. In: 2016 IEEE 16th International Conference on Data Mining Workshops (ICDMW). IEEE; 2016. https://doi. org/10.1109/icdmw.2016.0030.

12. Bradley PS, Lago-Peñas C, Rey E. Evaluation of the match performances of substitution players in elite soccer. Int J Sports Physiol Perform. 2014;9(3):415-24.

13. Di Mascio M, Bradley PS. Evaluation of the most intense high-intensity running period in english FA premier league soccer matches. J Strength Cond Res. 2013;27(4):909-15.

14. Carling C, Bradley P, McCall A, Dupont G. Match-to-match variability in high-speed running activity in a professional soccer team. J Sports Sci. 2016;34(24):2215-23.

15. Ingebrigtsen J, Dalen T, Hjelde GH, Drust B, Wisløff U. Acceleration and sprint profiles of a professional elite football team in match play. EJSS. 2015;15(2):101-10.

16. Varley MC, Aughey RJ. Acceleration profiles in elite australian soccer. Int $J$ Sports Med. 2013;34(1):34-9.

17. Vigne G, Gaudino C, Rogowski I, Alloatti G, Hautier C. Activity profile in elite italian soccer team. Int J Sports Med. 2010;31(5):304-10.

18. Andrzejewski M, Chmura J, Pluta B, Kasprzak A. Analysis of Motor Activities of Professional Soccer Players. 2012;26(6):1481-8. https://doi. org/10.1519/jsc.0b013e318231ab4c.

19. Castellano J, Blanco-Villaseñor A, Alvarez D. Contextual variables and time-motion analysis in soccer. Int J Sports Med. 2011;32(6):415-21.

20. Dalen T, Ingebrigtsen J, Ettema G, Hjelde GH, Wisløff U. Player load, acceleration, and deceleration during Forty-Five competitive matches of elite soccer. J Strength Cond Res. 2016;30(2):351-9.

21. Bradley PS, Carling C, Gomez Diaz A, Hood P, Barnes C, Ade J, Boddy M, Krustrup P, Mohr M. Match performance and physical capacity of players in the top three competitive standards of english professional soccer. Hum Mov Sci. 2013;32(4):808-21.

22. Di Salvo V, Gregson W, Atkinson G, Tordoff P, Drust B. Analysis of high intensity activity in premier league soccer. Int J Sports Med. 2009;30(3): 205-12.

23. Di Salvo V, Pigozzi F, González-Haro C, Laughlin M, De Witt J. Match performance comparison in top english soccer leagues. Int J Sports Med. 2012;34(06):526-32.

24. Bradley PS, Archer DT, Hogg B, Schuth G, Bush M, Carling C, Barnes C. Tier-specific evolution of match performance characteristics in the english premier league: it's getting tougher at the top. J Sports Sci. 2016;34(10):980-7.

25. Bush M, Barnes C, Archer DT, Hogg B, Bradley PS. Evolution of match performance parameters for various playing positions in the english premier league. Hum Mov Sci. 2015;39:1-11.

26. Rampinini E, Impellizzeri FM, Castagna C, Coutts AJ, Wisløff U. Technical performance during soccer matches of the italian serie a league: effect of fatigue and competitive level. J Sci Med Sport. 2009;12(1):227-33.

27. Russell M, Sparkes W, Northeast J, Cook CJ, Love TD, Bracken RM, Kilduff LP. Changes in acceleration and deceleration capacity throughout professional soccer Match-Play. J Strength Cond Res. 2016;30(10):2839-44.

28. Casamichana D, Castellano J, Castagna C. Comparing the physical demands of friendly matches and small-sided games in semiprofessional soccer players. J Strength Cond Res. 2012;26(3):837-43.

29. Hodgson C, Akenhead R, Thomas K. Time-motion analysis of acceleration demands of $4 \mathrm{v} 4$ small-sided soccer games played on different pitch sizes. Hum Mov Sci. 2014:33:25-32.

30. Mugglestone C, Morris JG, Saunders B, Sunderland C. Half-time and high-speed running in the second half of soccer. Int J Sports Med. 2013:34(9):847-8.

31. Bendiksen M, Pettersen SA, Ingebrigtsen J, Randers MB, Brito J, Mohr M Bangsbo J, Krustrup P. Application of the Copenhagen Soccer Test in high-level women players - locomotor activities, physiological response and sprint performance. 2013;32(6):1430-42. https://doi.org/10.1016/j. humov.2013.07.011.

32. Varley MC, Jaspers A, Helsen WF, Malone JJ. Methodological considerations when quantifying High-Intensity efforts in team sport using global positioning system technology. Int J Sports Physiol Perform. 2017;12(8):1059-68.

33. Harley JA, Lovell RJ, Barnes CA, Portas MD, Weston M. The interchangeability of global positioning system and semiautomated video-based performance data during elite soccer match play. J Strength Cond Res. 2011;25(8):2334-6. 
34. Fernandes-da-Silva J, Castagna C, Teixeira AS, Carminatti L, Guglielmo LGA. The peak velocity derived from the carminatti test is related to physical match performance in young soccer players. J Sports Sci. 2016;34(24):2238-45.

35. Hill-Haas SV, Rowsell GJ, Dawson BT, Coutts AJ. Acute physiological responses and time-motion characteristics of two small-sided training regimes in youth soccer players. J Strength Cond Res. 2009;23(1):111-5.

36. Abade EA, Gonçalves BV, Leite NM, Sampaio JE. Time-motion and physiological profile of football training sessions performed by under-15, under-17 and under-19 elite portuguese players. Int I Sports Physiol Perform. 2014;9(3):463-70.

37. Abade EA, Gonçalves BV, Silva AM, Leite NM, Castagna C, Sampaio JE. Classifying young soccer players by training performances. Percept Mot Skills. 2014;119(3):971-84.

38. Goto H, Morris JG, Nevill ME. Match analysis of U9 and U10 english premier league academy soccer players using a global positioning system: Relevance for talent identification and development. J Strength Cond Res. 2015;29(4):954-63.

39. Buchheit M, Mendez-Villanueva A, Simpson BM, Bourdon PC. Match running performance and fitness in youth soccer. Int J Sports Med. 2010;31(11):818-25.

40. Castagna C, Impellizzeri F, Cecchini E, Rampinini E, Alvarez JCB. Effects of intermittent-endurance fitness on match performance in young male soccer players. J Strength Cond Res. 2009;23(7):1954-9.

41. Goto H, Morris JG, Nevill ME. Motion analysis of U11 to U16 elite english premier league academy players. J Sports Sci. 2015;33(12):1248-58.

42. Buchheit M, Horobeanu C, Mendez-Villanueva A, Simpson BM, Bourdon PC. Effects of age and spa treatment on match running performance over two consecutive games in highly trained young soccer players. J Sports Sci. 2011;29(6):591-8.

43. Nagahara R, Morin J-B, Koido M. Impairment of sprint mechanical properties in an actual soccer match: A pilot study. Int I Sports Physiol Perform. 2016;11(7):893-8.

44. Akenhead R, Harley JA, Tweddle SP. Examining the external training load of an english premier league football team with special reference to acceleration. J Strength Cond Res. 2016;30(9):2424-32.

45. IFAB. Amendments to the Laws of the Game - 2015/2016. 2015;1-4. https://resources.fifa.com/mm/document/affederation/ifab/02/60/91/ 38/circular_log_amendments_2015_v1.0_en_neutral.pdf. Accessed 15 Apr 2019.

\section{Publisher's Note}

Springer Nature remains neutral with regard to jurisdictional claims in published maps and institutional affiliations.

Ready to submit your research? Choose BMC and benefit from:

- fast, convenient online submission

- thorough peer review by experienced researchers in your field

- rapid publication on acceptance

- support for research data, including large and complex data types

- gold Open Access which fosters wider collaboration and increased citations

- maximum visibility for your research: over $100 \mathrm{M}$ website views per year

At $\mathrm{BMC}$, research is always in progress.

Learn more biomedcentral.com/submissions 